\title{
Levetiracetam versus phenytoin for seizure prophylaxis in severe traumatic brain injury
}

\author{
Kristen E. Jones, M.D., ${ }^{1}$ Ava M. Puccio, R.N., Ph.D., ${ }^{1}$ Kathy J. Harshman, R.N., B.S.N., ${ }^{1}$ \\ Bonnie Falcione, Pharm.D., ${ }^{2}$ Neal Benedict, Pharm.D., ${ }^{2}$ Brian T. Jankowitz, M.D., 1 \\ Martina Stippler, M.D., ${ }^{1}$ Michael Fischer, B.S., ${ }^{1}$ Erin K. Sauber-Schatz, M.P.H., ${ }^{1}$ \\ Anthony Fabio, Ph.D., M.P.H., ${ }^{1}$ Joseph M. Darby, M.D., ${ }^{3}$ \\ AND David O. OKonkwo, M.D., PH.D. ${ }^{1}$
}

${ }^{1}$ Department of Neurological Surgery, University of Pittsburgh Medical Center; and Departments of ${ }^{2}$ Pharmacology and ${ }^{3}$ Critical Care Medicine, University of Pittsburgh, Pennsylvania

\begin{abstract}
Object. Current standard of care for patients with severe traumatic brain injury (TBI) is prophylactic treatment with phenytoin for 7 days to decrease the risk of early posttraumatic seizures. Phenytoin alters drug metabolism, induces fever, and requires therapeutic-level monitoring. Alternatively, levetiracetam (Keppra) does not require serum monitoring or have significant pharmacokinetic interactions. In the current study, the authors compare the EEG findings in patients receiving phenytoin with those receiving levetiracetam monotherapy for seizure prophylaxis following severe TBI.

Methods. Data were prospectively collected in 32 cases in which patients received levetiracetam for the first 7 days after severe TBI and compared with data from a historical cohort of 41 cases in which patients received phenytoin monotherapy . Patients underwent 1-hour electroencephalographic (EEG) monitoring if they displayed persistent coma, decreased mental status, or clinical signs of seizures. The EEG results were grouped into normal and abnormal findings, with abnormal EEG findings further categorized as seizure activity or seizure tendency.

Results. Fifteen of 32 patients in the levetiracetam group warranted EEG monitoring. In 7 of these 15 cases the results were normal and in 8 abnormal; 1 patient had seizure activity, whereas 7 had seizure tendency. Twelve of 41 patients in the phenytoin group received EEG monitoring, with all results being normal. Patients treated with levetiracetam and phenytoin had equivalent incidence of seizure activity $(\mathrm{p}=0.556)$. Patients receiving levetiracetam had a higher incidence of abnormal EEG findings $(\mathrm{p}=0.003)$.

Conclusions. Levetiracetam is as effective as phenytoin in preventing early posttraumatic seizures but is associated with an increased seizure tendency on EEG analysis. (DOI: 10.3171/FOC.2008.25.10.E3)
\end{abstract}

KEY WORDS $\bullet \quad$ antiepileptic drug $\bullet \quad$ posttraumatic seizure
seizure prophylaxis $\bullet \quad$ traumatic brain injury

$\mathrm{C}$ URRENT standard practice for patients with severe TBI is prophylactic treatment with phenytoin for 7 days after injury to decrease the risk of early posttraumatic seizures. ${ }^{3,11}$ Compared with placebo, phenytoin administration is associated with a relative risk of 0.25 for the occurrence of seizures during this time period (95\% CI $0.11-0.57) .{ }^{14}$ However, phenytoin therapy after the initial 7-day period has not been shown to reduce the development of late seizures nor has prophylaxis against

\footnotetext{
Abbreviations used in this paper: $\mathrm{AED}=$ antiepileptic drug; EEG = electroencephalographic; GCS = Glasgow Coma Scale; GOS = Glasgow Outcome Scale; $\mathrm{ICU}=$ intensive care unit; $\mathrm{TBI}=$ traumatic brain injury.
}

early seizures been shown to minimize morbidity or mortality rates associated with severe TBI. ${ }^{1,14}$

Phenytoin has a well-described side-effect profile that includes severe cutaneous hypersensitivity reactions and induction of the hepatic cytochrome P450 system, causing significant drug-drug interactions. ${ }^{5,10}$ Additionally, phenytoin usage requires close monitoring to maintain a narrow therapeutic window and has been described as causing fever and decreased levels of consciousness, particularly concerning in patients with TBI. For these reasons, alternative AED therapy has been sought. Valproate and carbamazepine have been investigated for usage in TBI but have similar side-effect profiles and require serum monitoring. 4.13 
K. E. Jones et al.

TABLE 1: Patient demographics—gender, age, and admission GCS stratified by cohort*

\begin{tabular}{|c|c|c|c|c|c|}
\hline \multirow[b]{2}{*}{ Variable } & \multicolumn{2}{|c|}{ Cohort } & \multirow[b]{2}{*}{ Chi-Square } & \multirow[b]{2}{*}{$d f$} & \multirow[b]{2}{*}{ p-Value } \\
\hline & Phenytoin & Levetiracetam & & & \\
\hline no. of patients & 12 & 15 & & & \\
\hline \multicolumn{6}{|l|}{ sex } \\
\hline$F$ & $3(25)$ & $4(27)$ & 0.010 & 1 & 0.922 \\
\hline M & $9(75)$ & $11(73)$ & & & \\
\hline \multicolumn{6}{|l|}{ age in yrs } \\
\hline$\leq 25$ & $4(33)$ & $4(27)$ & 1.89 & 3 & 0.596 \\
\hline $26-35$ & $2(16)$ & $6(40)$ & & & \\
\hline $36-45$ & $3(25)$ & $2(13)$ & & & \\
\hline$>46$ & $3(25)$ & $3(20)$ & & & \\
\hline \multicolumn{6}{|l|}{ GCS Score } \\
\hline $6-8$ & $5(42)$ & $10(67)$ & 1.688 & 1 & 0.194 \\
\hline $3-5$ & $7(58)$ & $5(33)$ & & & \\
\hline
\end{tabular}

Levetiracetam (Keppra, UCB, Inc.) is a non-enzyme-inducing AED that does not require serum level monitoring or induce fever or cutaneous hypersensitivity reactions and is not known to have significant pharmacokinetic interactions. ${ }^{7}$ In November 2006, FDA approval of the intravenous form of levetiracetam created an attractive option for antiseizure prophylaxis. Szaflarski et al. ${ }^{12}$ retrospectively analyzed levetiracetam use in a neuroscience ICU population (including patients with TBI, tumor, subarachnoid hemorrhage, stroke, and/or infection), and concluded that levetiracetam monotherapy was associated with lower complication rates and shorter ICU stays than treatment with other AEDs. To date, there are no published data investigating the efficacy of seizure prevention with levetiracetam compared with standard treatment with phenytoin in patients with severe TBI.

In the current study, we evaluated the occurrence of early posttraumatic seizure activity recorded by electroencephalography in patients with severe TBI treated with phenytoin versus levetiracetam as seizure prophylaxis. We hypothesized that levetiracetam would be equivalent to phenytoin in preventing early seizures in severe TBI. Secondarily, we hypothesized that in terms of abnormal EEG findings there would be no difference between patients treated with levetiracetam and those treated with phenytoin.

\section{Methods}

Under an innovative off-label investigational use policy approved by the Pharmacy and Therapeutics Committee at the University of Pittsburgh Medical Center, we initiated a protocol of intravenous levetiracetam monotherapy for early seizure prophylaxis in patients with severe TBI, defined by a postresuscitation GCS score of $3-8$. Levetiracetam therapy was initiated within 24 hours of injury, either in the emergency department or upon admission to the NeuroTrauma ICU.

From November 2006 to December 2007, 32 consec- utive patients with severe TBI were admitted and received levetiracetam $500 \mathrm{mg}$ IV every 12 hours for the first 7 days after traumatic injury. We then compared this prospective cohort to a historical cohort of patients from our severe TBI database in which patients received phenytoin for 7 days after trauma. From July 2005 to June 2006, 41 patients with severe TBI received IV phenytoin therapy within 24 hours of traumatic injury.

In our institution, all cases of severe TBI are managed in accordance with a standard protocol. Patients undergo an EEG examination if there is a suspicion of a seizure on the basis of mental status changes or persistent coma or if clinical seizure activity has occurred. In the current study, only patients who received an EEG examination were included in the analysis. Fifteen $(46.9 \%)$ of the 32 patients in the levetiracetam cohort warranted EEG testing to assess for seizures given persistent coma, change in mental status, or clinical seizure activity. Twelve (29.3\%) of the 41 patients in the phenytoin cohort required an EEG examination to investigate for seizures. Propofol is the sedative of choice in our TBI protocol and patients were weaned from propofol as tolerated at the time of EEG recordings per institutional protocol.

To assess the efficacy of seizure prevention in the 2 cohorts, individual EEG recordings were reviewed by an attending neurologist specializing in electroencephalography. The EEG findings for each patient were then stratified as normal or abnormal based on the presence of focal abnormal waveforms. Abnormal EEG findings were further classified into the following 3 categories: status epilepticus, seizure activity, or seizure tendency. The electroencephalograms categorized as demonstrating seizure tendency exhibited epileptiform activity of intermittent sharp waves or periodic lateralized epileptiform discharges without capturing electrographic seizures. When a patient underwent more than one EEG examination within the 7-day monitoring period, the most abnormal result was used for categorical stratification for that patient. 
Levetiracetam versus phenytoin for seizure prophylaxis

TABLE 2: Reason for EEG monitoring, imaging findings, and GOS scores in patients in the levetiracetam and phenytoin cohorts ${ }^{*}$

\begin{tabular}{|c|c|c|c|c|c|c|c|c|c|}
\hline $\begin{array}{l}\text { Cohort \& } \\
\text { Study ID }\end{array}$ & $\begin{array}{c}\text { Reason } \\
\text { for } \\
\text { EEG† }\end{array}$ & $\begin{array}{c}\text { CT } \\
\text { Score } \neq\end{array}$ & EDH & SDH & $\mathrm{ICH}$ & $\mathrm{SAH}$ & $\begin{array}{l}\text { Evac } \\
\text { of Mass } \\
\text { Lesion }\end{array}$ & $\begin{array}{l}\text { 3-Mo GOS } \\
\text { Score§ }\end{array}$ & $\begin{array}{l}\text { 6-Mo } \\
\text { GOS } \\
\text { Score }\end{array}$ \\
\hline \multicolumn{10}{|l|}{$\begin{array}{l}\text { levetira- } \\
\text { cetam }\end{array}$} \\
\hline $\mathrm{K} 5$ & $A$ & III & & & & $x$ & - & 1 & 1 \\
\hline K7 & A & $\|$ & $x$ & & $x$ & & - & 4 & 5 \\
\hline K9 & B & V & & $x$ & & & PTD 0 & 3 & 3 \\
\hline K11 & C & III & & & $x$ & $x$ & PTD 0 & 3 & 3 \\
\hline K12 & $A$ & I & & & & & - & 4 & 4 \\
\hline K13 & A & III & & & $x$ & $x$ & - & 1 & 1 \\
\hline K15 & C & III & & $x$ & $x$ & $x$ & - & 1 & 1 \\
\hline K16 & A & V & $x$ & & $x$ & $x$ & PTD 0 & 2 & 2 \\
\hline K18 & A & IV & & $x$ & $x$ & $x$ & - & 3 & 5 \\
\hline K20 & B & V & & $x$ & & & PTD 1 & 3 & 3 \\
\hline K23 & A & $\|$ & & $x$ & $x$ & $x$ & - & 3 & 3 \\
\hline K28 & A & V & & $x$ & & $x$ & PTD 0 & 1 & 1 \\
\hline K29 & B & V & $x$ & & & & PTD 0 & 4 & - \\
\hline K30 & $A$ & III & $x$ & & $x$ & $x$ & - & 1 & 1 \\
\hline К32 & A & V & $x$ & & & & PTD 0 & 3 & 3 \\
\hline median & & III & & & & & & 3 & 3 \\
\hline \multicolumn{10}{|l|}{$\begin{array}{l}\text { pheny- } \\
\text { toin }\end{array}$} \\
\hline D5 & $A$ & V & & $x$ & $x$ & $x$ & PTD 0 & 3 & 4 \\
\hline D7 & C & III & & & & $x$ & - & 1 & 1 \\
\hline D10 & $A$ & III & & & $x$ & $x$ & - & 1 & 1 \\
\hline D18 & A & $\|$ & & & $x$ & $x$ & - & 5 & 5 \\
\hline D19 & $A$ & V & $x$ & $x$ & $x$ & $x$ & PTD 0 & 4 & 4 \\
\hline $\mathrm{D} 20$ & $A$ & V & & $x$ & $x$ & $x$ & PTD 1 & 3 & 3 \\
\hline $\mathrm{D} 25$ & A & V & & $x$ & $x$ & & PTD 0 & 3 & 3 \\
\hline D29 & C & III & & & $x$ & $x$ & - & 2 & 2 \\
\hline D33 & $A$ & I & & & & & - & 2 & 3 \\
\hline D34 & A & III & & $x$ & $x$ & $x$ & - & 1 & 1 \\
\hline D39 & C & $\|$ & & & $x$ & $x$ & - & 2 & - \\
\hline D41 & B & V & & $x$ & $x$ & $x$ & PTD 0 & 3 & 3 \\
\hline median & & III & & & & & & 3 & 3 \\
\hline
\end{tabular}

* $\mathrm{EDH}=$ epidural hematoma; Evac = surgical evacuation; $\mathrm{SDH}=$ subdural hematoma; $\mathrm{ICH}$ = intracerebral hematoma; PTD = post-trauma day; SAH = subarachnoid hemorrhage; $x=$ present; $-=$ procedure not performed or data not obtained.

$\dagger$ Reason for EEG monitoring: $A$ = persistent comatose state; $B$ = decrease in level of consciousness; $\mathrm{C}=$ suspicion for clinical seizure activity.

₹ Classification according to Marshall et al., 1992: I = no visible intracranial abnormality; $\|$ = cisterns present, midline shift $0-5 \mathrm{~mm}$, no mass lesion $>25 \mathrm{~cm}^{3}$; III = cisterns compressed or absent, midline shift 0-5 $\mathrm{mm}$, no mass lesion > $25 \mathrm{~cm}^{3}$; IV = midline shift $>5 \mathrm{~mm}$, no mass lesion $>25 \mathrm{~cm}^{3} ; \mathrm{V}=$ evacuated mass lesion, any lesion surgically evacuated.

$\S$ Glasgow Outcome Scale scoring: 1 = dead; 2 = vegetative state; 3 = severe disability; 4 = moderate disability; 5 = good recovery.

The CT-based classification of head injury of Marshall et al. ${ }^{6}$ was used to define intracranial pathology on admission head CTs for each patient undergoing EEG monitoring. Glasgow Outcome Scale scores were obtained at 3 and 6 months posttrauma when possible.

Descriptive statistics, chi-square tests, and Mann- 
Whitney U-tests were used to compare the phenytoin and levetiracetam cohorts with respect to gender, age, and GCS score at admission. The Fisher exact test was used to determine whether there were significant differences between the 2 cohorts with respect to abnormal EEG findings, seizure activity, and seizure tendency with epileptiform activity. Analyses were performed in SPSS 15.0 for Windows (SPSS, Inc.). Statistical analysis of GOS scores obtained at 3 and 6 months postinjury was performed to monitor functional TBI outcomes.

\section{Results}

Univariate analysis was performed on possible confounders to test whether the differences seen between the drugs could be attributed to other factors. There were no significant differences in age, sex, or admission GCS scores between the cohorts (Table 1). Indication for obtaining an EEG, Marshall CT Classification, CT findings, and surgical interventions are outlined in Table 2. The median Marshall CT scores were the same in the 2 cohorts, and there was no statistically significant betweengroups difference with respect to the CT findings or frequency of craniotomy for evacuation of mass lesions.

Four of the patients in the levetiracetam cohort underwent 2 EEG studies, for a total of 19 EEG examinations performed in the 15 patients treated with levetiractam. Seven $(46.7 \%)$ of these 15 had normal EEG findings while $8(53.3 \%)$ had abnormal EEGs (Table 3). The abnormal EEG findings revealed no status epilepticus, although 1 $(12.5 \%)$ of the 8 patients with abnormal EEG findings had seizure activity, and $7(87.5 \%)$ had seizure tendency with abnormal waveforms (Table 3).

Four of the patients in the phenytoin cohort underwent 2 EEG studies and 1 underwent 3; thus a total of 19 EEG examinations were performed in the 12 patients treated with phenytoin. All 12 patients had normal findings (Table 3). The Fisher exact test showed a significant difference between the occurrence of abnormal EEG findings (seizure or seizure tendency with epileptiform activity) in the levetiracetam versus phenytoin cohorts $(\mathrm{p}=0.003)$, but there was no significant difference between the finding of seizures on electroencephalograms between the 12 phenytoin and 15 levetiracetam patients who underwent EEG monitoring ( $\mathrm{p}=0.556)$.

Although the study was not powered to detect outcome differences, GOS scores were collected at 3 and 6 months post injury (Table 2). We dichotomized the GOS scores to poor and good outcome $(1-3=$ poor; $4-5=$ good). With respect to GOS scores at 3 and 6 months, there were no statistically significant differences between patients treated with levetiracetam and those treated with phenytoin. Specifically, the chi-square statistic was 0.049 for GOS score at 3 months $(\mathrm{p}=0.825)$ and 0.115 for GOS score at 6 months $(\mathrm{p}=0.734)$. No patients were lost to follow-up at 3 months; one patient from each group was lost to follow-up at 6 months.

\section{Discussion}

The present study indicates that levetiracetam mono-
TABLE 3: Electroencephalographic findings stratified by cohort

\begin{tabular}{|c|c|c|c|c|c|}
\hline \multirow[b]{2}{*}{ EEG Finding } & \multicolumn{2}{|c|}{ Phenytoin } & \multicolumn{2}{|c|}{ Levetiracetam } & \multirow[b]{2}{*}{$p$ Value } \\
\hline & Yes & No & Yes & No & \\
\hline seizure tendency $\dagger$ & 0 & 12 & 7 & 8 & 0.007 \\
\hline seizure activity & 0 & 12 & 1 & 14 & 0.556 \\
\hline abnormal EEG & 0 & 12 & 8 & 7 & 0.003 \\
\hline
\end{tabular}

* Values represent numbers of patients unless otherwise indicated. † Seizure tendency included epileptiform activity of intermittent sharp waves or periodic lateralized epileptiform discharges without electrographic seizures.

therapy in the first 7 days following severe TBI is associated with an increased seizure tendency and increased epileptiform activity on electroencephalograms compared with phenytoin. The rates of seizure activity were equivalent in patients treated with levetiracetam and those treated with phenytoin. The implications of increased seizure tendency and epileptiform activity require further study.

Traumatic brain injury occurs in approximately 1.5 million people in the US each year. ${ }^{9}$ One important complication of TBI is seizure. Seizure risk after TBI is related to injury severity. In one study, ${ }^{1}$ seizures developed during the first year postinjury in $<1 \%$ of patients with mild TBI and in $6 \%$ of patients with severe TBI. Temkin et al. ${ }^{14}$ found a 2 -year seizure rate of $21 \%$ in patients with severe TBI. Early seizures, defined as occurring within the first 7 days posttrauma, have been shown to increase intracranial pressure episodically, thereby increasing the risk for cerebral ischemia. ${ }^{15}$ Late seizures, developing after the initial 7-day period, are associated with a worse functional outcome measured by the GOS. ${ }^{2}$ Early seizure prophylaxis, however, does not influence occurrence of late seizures.

Ronne-Engstrom and Winkler, ${ }^{2}$ in study of a series of cases involving continuous EEG recordings in patients with unspecified severity of TBI, reported high-frequency bursts of epileptiform activity that developed into electrographic seizures in $12(66.7 \%)$ of 18 patients. ${ }^{8}$ The current study is limited by lack of 24-hour EEG monitoring, raising the possibility of the occurrence of seizures not captured by 1-hour EEG monitoring. Patients in our study treated with levetiracetam whose EEG studies showed seizure tendency may have had uncaptured waveform deterioration into electrographic seizures. The current study is limited further by a small sample size and by the retrospective, historical cohort design.

The strength of the current findings lies in the use of actual EEG data to evaluate brain and seizure activity in relation to the use of AEDs as seizure prophylaxis following severe TBI. We restricted the analysis to patients in whom EEG monitoring was performed because overt 


\section{Levetiracetam versus phenytoin for seizure prophylaxis}

clinical seizures themselves are relatively rare events and subject to observer bias.

The current study highlights the fact that clinical seizures are difficult to identify through observation or physical examination in the early stages after severe TBI. Both the TBI itself and sedative and neuromuscular blockade agents used in intensive care management of severe TBI may mask seizure activity. Therefore, any prospective study intended to test the utility of levetiracetam versus phenytoin for seizure prophylaxis after severe TBI should include routine use of EEG monitoring to discern abnormal EEG patterns and between-group differences in seizure frequency.

Since levetiracetam does not require loading doses or monitoring of drug levels and lacks significant drug-drug interaction, it is an appealing alternative therapy in the prevention of early posttraumatic seizures. On the basis of the results of the current study, we urge caution against widespread practice changes in converting to levetiracetam as monotherapy for seizure prophylaxis following severe TBI. Prospective studies investigating levetiracetam in TBI are ongoing, although the results may require further scrutiny if objective data, like EEG recordings, are not included.

\section{Conclusions}

Our data indicate that levetiracetam is as effective as phenytoin in preventing early posttraumatic seizures. Nevertheless, levetiracetam monotherapy was associated with increased frequency of abnormal EEG findings. Further study is necessary to determine the clinical implication of this finding as well as to determine whether these patients exhibit a higher incidence of late posttraumatic seizures. Prospective studies comparing levetiracetam and phenytoin among subsets of patients with mild, moderate, and severe TBI are indicated. Because prophylaxis against early seizures has not been shown either to reduce the incidence of late posttraumatic seizures or influence outcome, future prospective studies should also include a placebo group to assess the possibility that early seizure prophylaxis following TBI exposes patients to unnecessary morbidities of AEDs without benefits of enhancing neurological recovery.

\section{Disclosure}

This research was performed at the University of Pittsburgh Medical Center and was supported by the University of Pittsburgh Brain Trauma Research Center (NIH P50NS30318).

\section{Disclaimer}

The authors report no conflict of interest concerning the materials or methods used in this study or the findings specified in this paper.

\section{References}

1. Annegers JF, Hauser WA, Coan SP, Rocca WA: A populationbased study of seizures after traumatic brain injuries. N Engl J Med 338:20-24, 1998

2. Asikainen I, Kaste M, Sarna S: Early and late posttraumatic seizures in traumatic brain injury rehabilitation patients: brain injury factors causing late seizures and influence of seizures on long-term outcome. Epilepsia 40:584-589, 1999

3. Chang BS, Lowenstein DH: Practice parameter: antiepileptic drug prophylaxis in severe traumatic brain injury: report of the Quality Standards Subcommittee of the American Academy of Neurology. Neurology 60:10-16, 2003

4. Glotzner FL, Haubitz I, Miltner F, Kapp G, Pflughaupt KW: [Seizure prevention using carbamazepine following severe brain injuries]. Neurochirurgia (Stuttg) 26:66-79, 1983 (Ger)

5. Jones GL, Wimbish GH, McIntosh WE: Phenytoin: basic and clinical pharmacology. Med Res Rev 3:383-434, 1983

6. Marshall LF, Marshall SB, Klauber MR, Van Berkum Clark M, Eisenberg H, Jane JA, et al: The diagnosis of head injury requires a classification based on computed axial tomography. J Neurotrauma 9 (Suppl 1):S287-S292, 1992

7. Ramael S, Daoust A, Otoul C, Toublanc N, Troenaru M, Lu ZS, et al: Levetiracetam intravenous infusion: a randomized, placebo-controlled safety and pharmacokinetic study. Epilepsia 47:1128-1135, 2006

8. Ronne-Engstrom E, Winkler T: Continuous EEG monitoring in patients with traumatic brain injury reveals a high incidence of epileptiform activity. Acta Neurol Scand 114:47-53, 2006

9. Rutland-Brown W, Langlois JA, Thomas KE, Xi YL: Incidence of traumatic brain injury in the United States, 2003. J Head Trauma Rehabil 21:544-548, 2006

10. Sahin S, Comert A, Akin O, Ayalp S, Karsidag S: Cutaneous drug eruptions by current antiepileptics: case reports and alternative treatment options. Clin Neuropharmacol 31:9396, 2008

11. Schierhout G, Roberts I: Anti-epileptic drugs for preventing seizures following acute traumatic brain injury. Cochrane Database Syst Rev 4: CD000173, 2001

12. Szaflarski JP, Meckler JM, Szaflarski M, Shutter LA, Privitera MD, Yates SL: Levetiracetam use in critically ill patients. Neurocrit Care 7:140-147, 2007

13. Temkin NR, Dikmen SS, Anderson GD, Wilensky AJ, Holmes MD, Cohen W, et al: Valproate therapy for prevention of posttraumatic seizures: a randomized trial. J Neurosurg 91:593-600, 1999

14. Temkin NR, Dikmen SS, Wilensky AJ, Keihm J, Chabal S, Winn HR: A randomized, double-blind study of phenytoin for the prevention of post-traumatic seizures. N Engl J Med 323:497-502, 1990

15. Vespa PM, Miller C, McArthur D, Eliseo M, Etchepare M, Hirt D, et al: Nonconvulsive electrographic seizures after traumatic brain injury result in a delayed, prolonged increase in intracranial pressure and metabolic crisis. Crit Care Med 35:2830-2836, 2007

Manuscript submitted June 15, 2008.

Accepted August 18, 2008.

Address correspondence to: David O. Okonkwo, M.D., Ph.D., Department of Neurological Surgery, University of Pittsburgh, 200 Lothrop Street, Suite B-400, Pittsburgh, Pennsylvania 15213. email: okonkwodo@upmc.edu. 